\title{
Characterization and enhancement of non-invasive recordings of intestinal myoelectrical activity
}

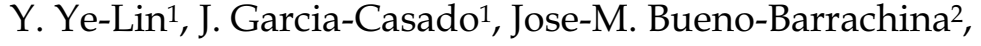 \\ J. Guimera-Tomas ${ }^{1}$, G. Prats-Boluda ${ }^{1}$ and J.L. Martinez-de-Juan ${ }^{1}$ \\ ${ }^{1}$ Instituto Interuniversitario de Investigación en Bioingeniería y Tecnología Orientada al \\ Ser Humano, Universidad Politécnica de Valencia, Spain \\ 2Instituto de Tecnología Eléctrica, Universidad Politécnica de Valencia, Spain
}

\section{Intestinal motility}

Intestinal motility is a set of muscular contractions, associated with the mixing, segmentation and propulsion actions of the chyme, which is produced along the small intestine (Weisbrodt 1987). Therefore, intestinal motility is basic for the process of digesting the chyme that is coming from the stomach.

Under physiological conditions, intestinal motility can be classified in two periods: fasting motility and postprandial motility. In the fasting state, the small intestine is not quiescent, but it is characterized by a set of organized contractions that form a pattern named Interdigestive Migrating Motor Complex (IMMC) (Szurszewski 1969). This pattern of contractile activity has a double mission: to empty the content that is being poured by the stomach and to prevent the migration of germs and bacteria in the oral way (Szurszewski 1969; Weisbrodt 1987). The IMMC has a length between 90 and 130 minutes in humans and between 80 and 120 minutes in dogs. Attending to the motor activity degree of the intestine, the IMMC cycle can be divided in three phases (Szurszewski 1969; Weisbrodt 1987): phase I of quiescence, which is characterized by the absence of contractile activity; phase II of irregular contractile activity; and phase III of maximal frequency and intensity of bowel contractions. Phase III is band of regular pressure waves lasting for about $5 \mathrm{~min}$ and migrates aborally from the proximal small intestine to the terminal ileum. It is usually generated at the duodenum, although it can be generated at any point between the stomach and the ileum. Migration is a prerequisite for the phase III. The velocity of migration is $5-10 \mathrm{~cm} / \mathrm{min}$ in the proximal small intestine and it decreases gradually along the small intestine to $0.5-1 \mathrm{~cm} / \mathrm{min}$ in the ileum (Szurszewski 1969; Weisbrodt 1987). The IMMC is cyclic at fast and it is interrupted after the food ingestion, which involves the appearance of the postpandrial motility. The postpandrial pattern is characterized by an irregular contractile activity similar to the phase II of the IMMC. In figure 1, it can be appreciated a complete IMMC cycle from minute 55 until minute 155, and the appearance of the postpandrial motility pattern occurred immediately after the ingestion of food. 


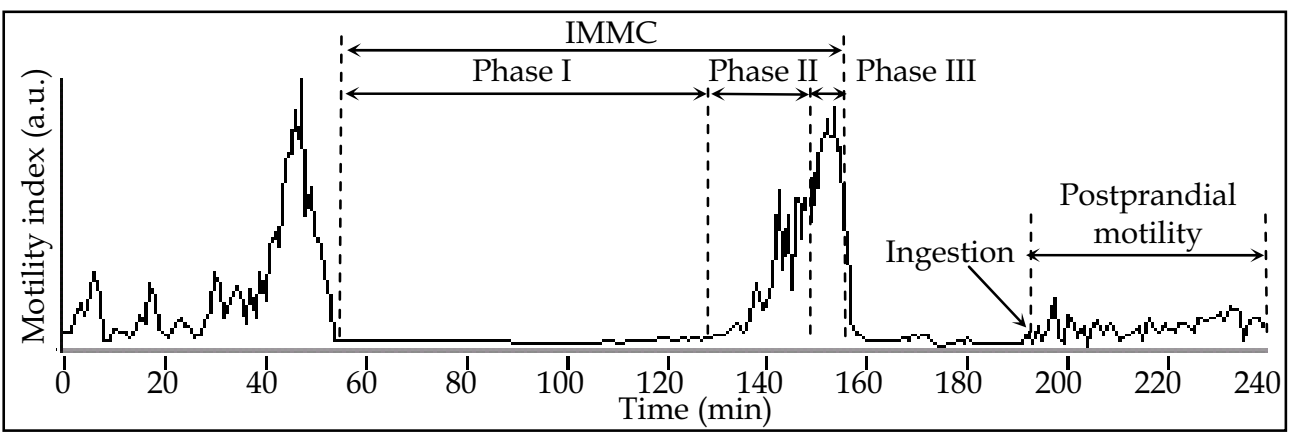

Fig. 1. Time evolution of intestinal motility index recorded from canine jejunum in fasting state and after ingestion (minute 190).

Many pathologies such as irritable bowel syndrome, mechanical obstruction, bacterial overgrowth or paralytic ileum are associated with intestinal motor dysfunctions (Camilleri et al. 1998; Quigley 1996). These dysfunctions show a high prevalence: between $10 \%$ and $20 \%$ of European and American population suffers from functional bowel disorders and irritable bowel syndrome (Delvaux 2003). Because of that, the study of the intestinal motility is of great clinical interest.

\section{Recording of intestinal motility}

The main problem in monitoring the intestinal activity is the anatomical difficult access to the small bowel. Traditionally, intestinal motility measurement has been performed by means of manometric techniques, because these are low cost techniques and they are a direct measurement of the intestinal contractions. However, this method presents a series of technical and physiological problems (Byrne \& Quigley 1997; Camilleri et al. 1998), and its non-invasiveness is still a controversial issue.

Nowadays, non-invasive techniques for the intestinal motility monitoring are being developed such as: ultrasound based techniques (An et al. 2001), intestinal sounds (Tomomasa et al. 1999), bioelectromagnetism based techniques (Bradshaw et al. 1997), and myoelectrical techniques (Bradshaw et al. 1997; Chen et al. 1993; Garcia-Casado et al. 2005). The utility of the intestinal sounds recording sounds so as to determinate the intestinal motility has been questioned, because it is better corresponded to the intestinal transit associated with the propulsion movements rather than to the intestinal contractions (Tomomasa et al. 1999). The ultrasound techniques have been validated for the graphical visualization and the quantitative analysis of both the peristaltic and non-peristaltic movements of the small intestine (An et al. 2001), but they do not closely represent the intestinal motility. On the other hand, both the myoelectrical and the magnetical studies have demonstrated the possibility of picking up the intestinal activity on the abdominal surface (Bradshaw et al. 1997), providing a very helpful tool for the study of the gastrointestinal motor dysfunctions. However, the clinical application of the magnetic techniques is limited by the high cost of the devices (Bradshaw et al. 1997), and the development of the myoelectrical techniques is still in the experimental stage. 
At the present chapter, the study of the intestinal activity is focused on the myoelectrical techniques. These techniques are based on the recording of the changes of muscular cell's membrane potential and the associated bioelectrical currents, since they are directly related to the small intestine smooth muscle contractions.

\section{Intestinal myoelectrical activity}

The electroenterogram (EEnG) is the myoelectrical intestinal signal originated by the muscular layers and it can be recorded on the intestinal serous wall. The EEnG is composed by two components: slow waves (SW), which is a pacemaker activity and does not represent the intestinal motility; and action potentials, also known as spike bursts (SB). These SB only appear at the plateau of the slow wave when the small intestine contracts, showing the presence and the intensity of the intestinal contraction (Martinez-de-Juan et al. 2000; Weisbrodt 1987). The relationship between the intestinal pressure and the SB activity is widely accepted (Martinez-de-Juan et al. 2000; Weisbrodt 1987). This relationship can be appreciated in figure 2, the presence of SB (trace b) is directly associated with the increments on the intestinal pressure (trace a). It can also be observed that the SW activity is always present, even when no contractions occur.

Nowadays, the hypothesis that the SW activity is generated by the interstitial cells of Cajal is widely accepted (Horowitz et al. 1999). These cells act as pacemaker cells since they possess unique ionic conductances that trigger the SW activity, whilst smooth muscle cells may lack the basic ionic mechanisms which are necessary to generate the SW activity (Horowitz et al. 1999). However, smooth muscle cells respond to the depolarization and repolarization cycle imposed by the interstitial cells of Cajal. The responses of smooth muscle cells are focused on the regulation of L-type $\mathrm{Ca}^{2+}$ current, which is the main source of $\mathrm{Ca}^{2+}$ that produce the intestinal contraction (Horowitz et al. 1999). Therefore, the frequency of the SW determines the maximal rhythm of the intestinal mechanical contraction (Weisbrodt 1987). The SWs are usually generated in the natural pacemaker that is localized at the duodenum, and they propagate from the duodenum to the ileum. The SW frequency is approximately constant at

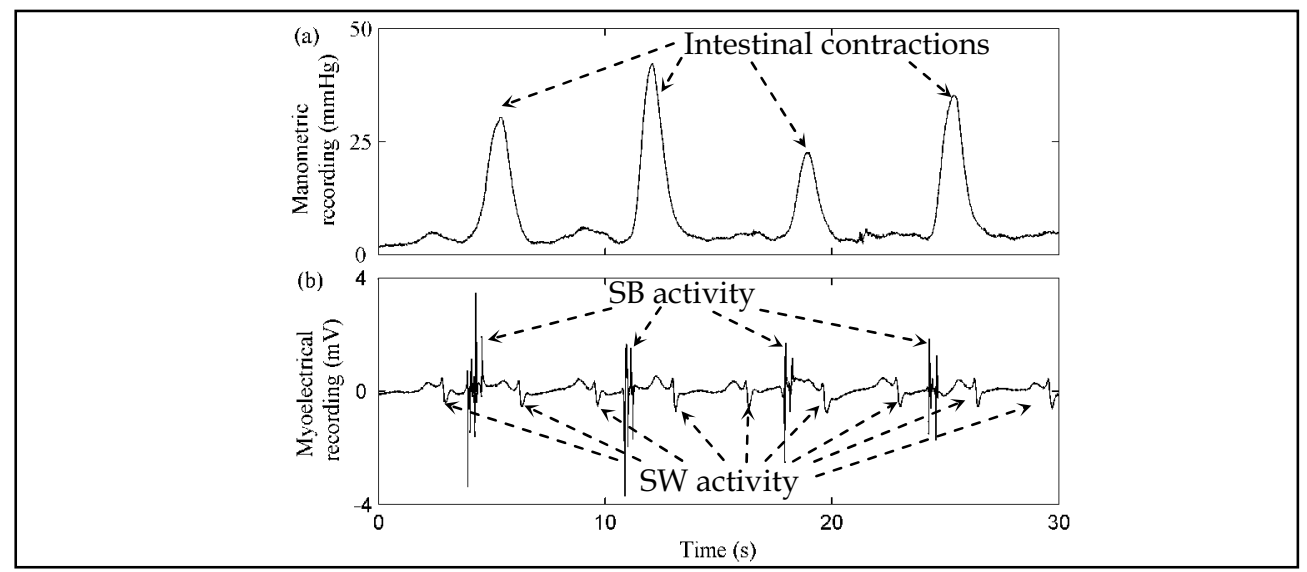

Fig. 2. Simultaneous recording of bowel pressure (a) and internal myoelectrical activity (b) in the same bowel loop from a non-sedated dog. 
each point of the intestine although it decreases in distal way (Diamant \& Bortoff 1969). In dogs this frequency ranges from approximately 19 cycles per minute (cpm) at the duodenum to $11 \mathrm{cpm}$ at the ileum (Bass \& Wiley 1965). In humans the SW frequency is around $12 \mathrm{cpm}$ at upper duodenum and of $7 \mathrm{cpm}$ at the terminal ileum.

With regard to the SB, they are generated by the smooth muscle cells which are responsible for the intestinal mechanical contraction (Horowitz et al. 1999). The smooth muscle of the small intestine is controlled by the enteric nervous system, and it is influenced by both the extrinsic autonomic nerves of the nervous system and the hormones (Weisbrodt 1987). Unlike the SW activity, the SB activity does not present a typical repetition frequency, but it is characterized for distributing its energy in the spectrum over $2 \mathrm{~Hz}$ in the internal recording of the EEnG (Martinez-de-Juan et al. 2000).

The internal recording of EEnG provides a signal of 'high' amplitude, i.e. in the order of $\mathrm{mV}$, which is almost free of physiological interferences. The employment of this technique has obtained promising results for the characterization of different pathologies such as: intestinal ischemia (Seidel et al. 1999), bacterial overgrowth in acute pancreatitis (Van Felius et al. 2003), intestinal mechanical obstruction (Lausen et al. 1988), irritable bowel syndrome (El-Murr et al. 1994). However, the clinical application of internal myoelectrical techniques is limited, given that surgical intervention is needed for the implantation of the electrodes.

\section{Surface EEnG recording}

Surface EEnG recording can be an alternative method to non-invasively determine the intestinal motility. Logically, the morphology and the frequency spectrum of the intestinal myoelectrical signals recorded on the abdominal surface are affected by the different abdominal layers, which exercise an insulating effect between the intestinal sources and the external electrodes (Bradshaw et al. 1997).

\subsection{Non-invasive recording and characterization of slow wave activity}

In 1975, in an experiment designed to measure the gastric activity using surface electrodes, Brown found a component of frequency of 10-12 cpm, superposed on $3 \mathrm{cpm}$ gastric electrical activity (Brown et al. 1975). They believed that the component of 10-12 cpm was of intestinal origin. Later, by means of the analysis of the simultaneous external and internal EEnG recordings, it was confirmed that it is possible to detect the intestinal SW on the human abdominal surface (Chen et al. 1993). In this last work, bipolar recording of surface signal was conducted using two monopolar contact electrodes which were placed near the umbilicus with a spacing distance of $5 \mathrm{~cm}$. Figure 3 shows 5 min of the external EEnG signal (electrodes 3-4), simultaneously recorded with the gastric activity (electrodes 1-2) and the respiration signal. The external EEnG signal presents an omnipresent frequency peak of 9-12 cpm, which coincides with the typical value of the repetition rate of the human intestinal SW (12 cpm at the duodenum and $7 \mathrm{cpm}$ at the ileum). The simultaneous recording of respiration signal allowed rejecting breathing as a possible source of this frequency peak.

The possibility of picking up the intestinal SW activity on the abdominal surface has been reasserted by other authors (Bradshaw et al. 1997; Chang et al. 2007; Garcia-Casado et al. 2005). The myoelectrical signal recorded on the abdominal surface of patients with total gastrectomy presented a dominant frequency of $10.9 \pm 1.0 \mathrm{cpm}$ in fasting state and $10.9 \pm 1.3 \mathrm{cpm}$ in postprandial state (Chang et al. 2007). In animal models it has been proven 


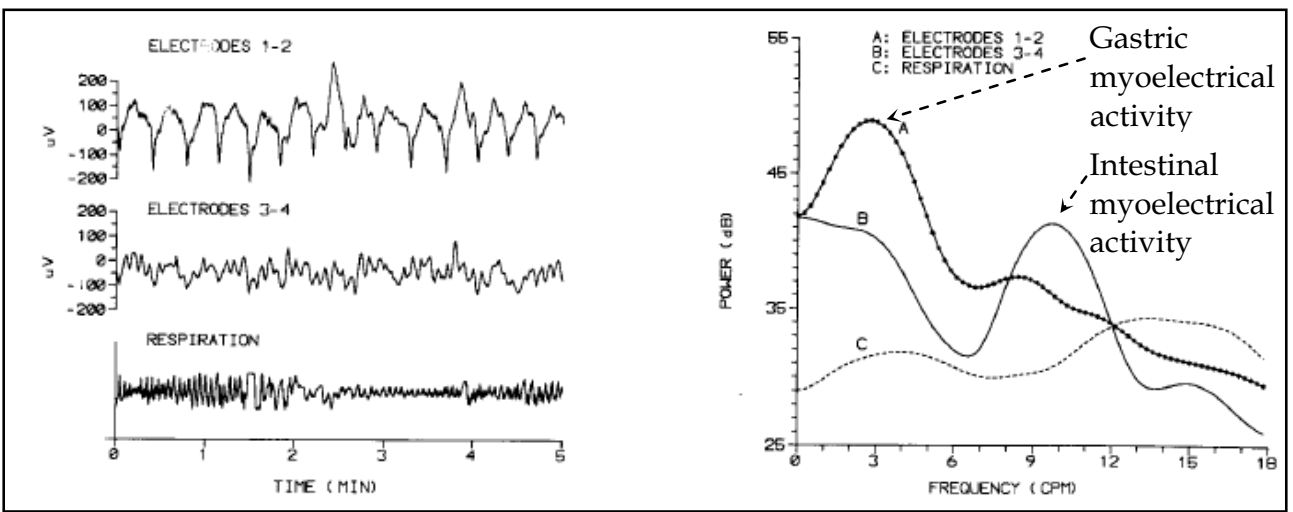

Fig. 3. Five minutes of external gastric (electrode 1-2) and intestinal (electrode 3-4) myoelectrical signal, simultaneously recorded with the respiration signal (bottom trace). The right trace shows the power spectral density of these signals (Chen et al. 1993).

that the dominant frequency of the external myoelectrical intestinal signal coincides with the repetition rate of the internal intestinal SW both in physiological conditions (Garcia-Casado et al. 2005) and in pathological conditions (Bradshaw et al. 1997).

Unlike the internal myoelectrical signal, the amplitude of the external record shows a great variation from 30 to $330 \mu \mathrm{V}$ among subjects (Chen et al. 1993), since this amplitude depends on a set of factors such as the body mass index of the subject and the recording conditions (preparation of the skin, the contact of the electrode with the skin and the distance from the source of activity). Some authors evaluated the reliability of the information contained in the external recording of the electrogastrogram (EGG), which is a very similar signal to the intestinal myoelectrical signal (Mintchev \& Bowes 1996). In that study, the following parameters of EGG signals were analyzed: the amplitude, the frequency, the time shift between different channels recorded simultaneously and the waveform. They concluded that the signal frequency is the unique consistent and trustworthy parameter of the external myoelectrical recording (Mintchev \& Bowes 1996). Because of that, the analysis of the SW activity of the external EEnG is usually focused on obtaining the dominant frequency of the signal, which allows determining the intestinal SW repetition rate.

To obtain the dominant frequency of the external EEnG signal, some researchers have used non-parametric spectral estimation techniques (Chen et al. 1993; Garcia-Casado et al. 2005). These studies have showed the utility of these techniques for the identification of the intestinal SW activity on the abdominal surface. By means of these non-parametric techniques it has also been determined that the energy associated with the intestinal SW is concentrated between 0.15 and $2 \mathrm{~Hz}$ in the animal model (Garcia-Casado et al. 2005). Nevertheless, these techniques present some disadvantages: the selection of the window length to be used in the analysis has an important repercussion on the frequency resolution and on the stationarity of the signal. Other authors proposed the use of parametric techniques based on autoregressive models (Bradshaw et al. 1997; Moreno-Vazquez et al. 2003; Seidel et al. 1999) or on autoregressive moving average models (Chen et al. 1990; Levy et al. 2001) to obtain the frequency of the external signal. The advantage of these techniques with respect to the non-parametric techniques is that they enable to determine the dominant 
frequency of the signal with better frequency resolution even with a shorter window of analysis. Nevertheless, the application of these techniques present some practical limitations: the information related to the power associated with each frequency is not trustworthy. In short, it is advisable to use parametric techniques in order to identify the peak frequencies of the signal, whereas if the aim is to study the energy distribution of the signal in the frequency domain, non-parametric spectral analysis is more appropriate.

\subsection{Non-invasive recording and characterization of spike bursts activity}

The first works that studied the possibility of recording the SB activity of gastrointestinal origin non-invasively, were conducted analyzing the gastric SW in the external recordings (Atanassova et al. 1995; Chen et al. 1994). They stated that the presence of the SB in the internal recordings increases the amplitude of the external gastric SW (Atanassova et al. 1995), and it also leads to an increase in the instability of the power of the dominant frequency associated with the external gastric SW (Chen et al. 1994). Nevertheless, these hypotheses were refuted by other authors, causing a great controversy (Mintchev \& Bowes 1996). They believed that the increase of the amplitude of the surface SW activity is due to the minor distance between the myoelectrical signal of origin and the surface electrodes associated with the stomach distension when the SB are present (Mintchev \& Bowes 1996), rather than being directly related to the contractile activity of the stomach.

Very few works about external recordings of gastrointestinal activity have focused their studies out of the SW frequency band (Akin \& Sun 1999; Garcia-Casado et al. 2005). In Akin's work, it was shown that the energy associated with gastric SB activity ranges from $50-80 \mathrm{cpm}$ by means of spectral analysis in an animal model (50-80 cpm) (Akin \& Sun 1999). The correlation study of the internal and external signal energy in that frequency range showed a high correlation index (around 0.8) (Akin \& Sun 1999). Regarding to the intestinal myoelectrical signal, only a few works have been found that study the two components of the surface electroenterogram (EEnG) and not only the SW intestinal activity (Garcia-Casado et al. 2005; Ye et al. 2008). In both works, it was carried out a comparative study of the internal and external recordings of intestinal myoelectrical signal from dogs. Bipolar external recording was obtained using two monopolar contact electrodes placed on the abdominal surface. Figure 4 shows the simultaneous recording of internal (top traces) and surface signals (bottom traces) in a period of rest and in a period of maximum contractile activity. In the period of rest, 9 slow waves in $30 \mathrm{~s}$ can be observed both in the internal and in the external recording. On the other hand, in the period of maximum contractile activity which corresponds to the phase III of the IMMC, in the internal recording it can be observed that every SW is accompanied by a superposed SB, whereas in the external recording a high frequency component of low amplitude is superposed to the SW activity (fig. 4 right, bottom trace). Since it is not synchronized with the cardiac activity, and the SB activity is the high frequency component of EEnG recording (Martinez-de-Juan et al. 2000), these high frequency components on the external EEnG recording are believed to be associated with the intestinal SB activity (Garcia-Casado et al. 2005).

In order to study the intestinal SB activity on the surface recording, time-frequency analysis have been proposed to obtain simultaneous information both on spectral content and on time intervals (Garcia-Casado et al. 2002). These studies showed that Choi-Williams distribution is the best time-frequency distribution in order to identify the presence of SB, 

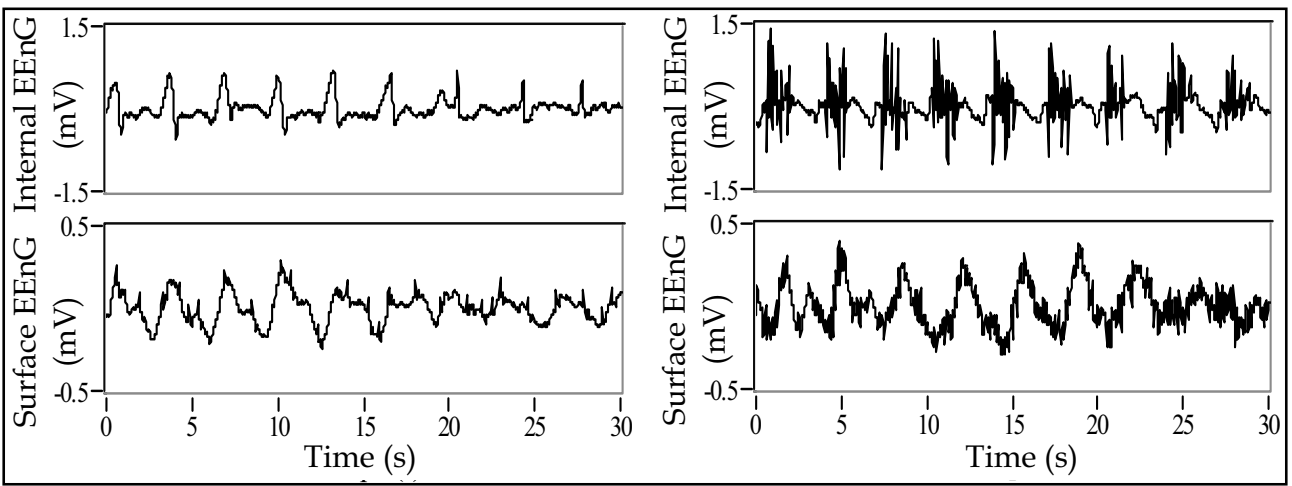

Fig. 4. Simultaneous recording of canine intestinal myoelectrical activity in fasting state during a period of rest (left traces) and during a period of maximum contractile activity (right traces). Signals are recorded in the intestinal serosa (top traces) and on abdominal surface (bottom traces) (Garcia-Casado et al. 2005).

whereas spectrogram is more useful in order to quantify the SB activity (Garcia-Casado et al. 2002). Other studies defend that non-parametric spectral techniques also can be used to study the external EEnG signal (Garcia-Casado et al. 2005), since it can be assumed the hypothesis of the stationarity of the signal if the size of the window is sufficiently small. Based on these non-parametric techniques, it has been shown that the energy of the intestinal SB activity of the external recording is concentrated between 2 and $20 \mathrm{~Hz}$ (GarciaCasado et al. 2005). Therefore, the energy in this frequency band of the external EEnG, also named as SB energy, could be of great utility to quantify in a non-invasive way the intestinal motor activity (Garcia-Casado et al. 2005).

Nevertheless, the study of Garcia-Casado presents certain limitations from the medical point of view: a segment of intestine was sutured to the internal abdominal wall so as to obtain a reference pattern for the intestinal activity of the external recording (Garcia-Casado et al. 2005). In spite of the fact that the small intestine has natural adherences to the abdominal internal wall, the above mentioned artificial attachment might improve the electrical contact between the surface electrodes and the intestine (Bradshaw et al. 1997). Therefore, it can be expected that the signal-to-interference ratio of the external recording would be decreased if this artificial attachment was eliminated. On the other hand, the elimination of the artificial attachment would also have another consequence: there is no longer knowledge of the intestinal segment whose activity is being picked up on the external recording.

The latest studies have focused their efforts on the comparison between the external and internal recording of the canine intestinal myoelectrical signal in fasting state, but without the artificial attachment of an intestinal segment to the internal abdominal wall (Ye et al. 2008). Figure 5 shows the evolution of the SB energy of the external recording (trace a) with the intestinal motility index (IMI) of the different internal channels (traces b-d) acquired simultaneously in fasting state. In these figures, it is possible to identify two complete cycles of the IMMC in the different internal channels. The SB energy in the external recording shows two periods of maximum intensity (about the minute 85 and minute 167), that are probably related to the periods of maximum contractile activity of the jejunum (in the 


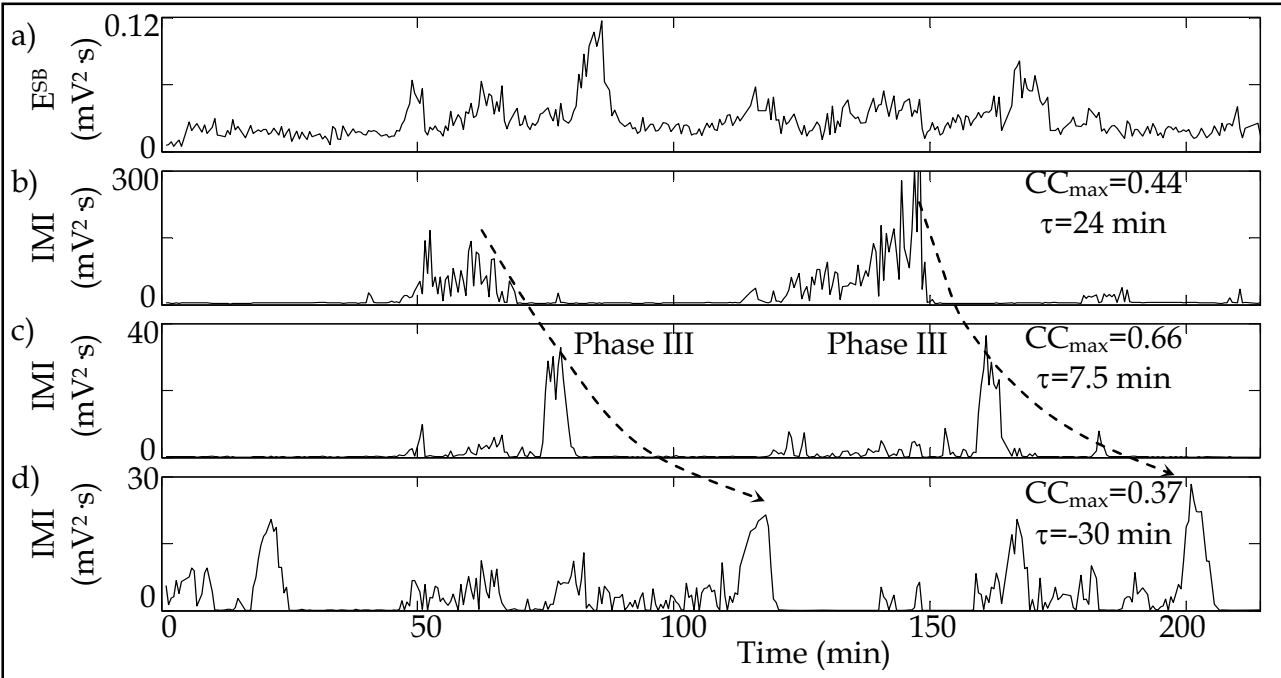

Fig. 5. Intestinal motility indicators of canine external and internal EEnG recording acquired simultaneously in fasting state: a) Surface. b) Duodenum. c) Jejunum. d) Ileum. It is also indicated the maximum value of the cross-correlation function $\left(\mathrm{CC}_{\max }\right)$ between the $\mathrm{SB}$ energy of external recording and the internal IMI and its corresponding time lag $\tau$.

minutes 78 and 160). This time lag is probably due to the disagreement of the recording area between the external and internal recordings. Since the phase III of the IMMC propagates in the distal way in fasting state, the external electrodes might be recording the intestinal activity from one segment of intestine located approximately $35 \mathrm{~cm}$ distally to the jejunum internal recording. In this context, the use of the cross-correlation function allows to make the adjustment of the possible delay, and thus reflect the relationship between the SB energy of the external recording with the internal IMI. In this case, the maximum value of the cross-correlation function (0.66) is obtained with the IMI of the jejunum channel when adjusting a delay of 7.5 minutes. The results of these preliminary studies confirm the possibility of picking up the intestinal SB activity on the abdominal surface recordings of the EEnG under physiological conditions without the need of artificial attachments (Ye et al. 2008). This means a great advance in the study of the intestinal motility by means of the non-invasive myoelectrical techniques.

\subsection{Limitations of external EEnG recording}

In the previous sections, it has been shown that both components of the intestinal myoelectrical activity can be recorded on the abdominal surface, and that spectral parameters are very useful to characterize these components: the dominant frequency of the signal to determine the frequency of the intestinal pacemaker, i.e. the SW; the SB energy to determine the intensity of the possible intestinal contractions. Nevertheless, the surface EEnG still presents some difficulties for its clinical application. First, the myoelectrical intestinal signal recorded on abdominal surface is a very small amplitude signal (Bradshaw et al. 1997; Chen et al. 1993; Garcia-Casado et al. 2005; Prats-Boluda et al. 2007), especially in 
the SB frequency range (Garcia-Casado et al. 2005), due to the insulating effect of the abdominal layers and to spatial filtering (Bradshaw et al. 1997). However, the major problem of the surface recording of the myoelectrical signal resides in the presence of strong interferences: electrocardiogram (ECG), respiration, movement artifacts, components of very low frequency and other interferences of minor relevancy (Chen et al. 1993; Garcia-Casado et al. 2005; Liang et al. 1997; Prats-Boluda et al. 2007; Verhagen et al. 1999). The presence of these interferences may impede the obtaining of trustworthy parameters derived from the external myoelectrical recordings which define the intestinal activity. This is a common problem in the non-invasive recording of the gastric, colonic, uterine and intestinal activities. In the case of the surface EEnG, the amplitude of these interferences can be of the same order of magnitude or even higher than the amplitude of the target signal. Consequently, the identification and the elimination of these interferences are of great importance in order to extract useful information from the surface EEnG. Next it is briefly described the different interferences that can appear in the surface EEnG recording:

- Electrocardiogram (ECG): ECG interference concerns principally the high frequency components of the external EEnG i.e. the SB, since the SB activity recorded on abdominal surface are of very low amplitude (Garcia-Casado et al. 2006). Conventional filters cannot be used for the elimination of ECG interference since its spectrum is overlapped with that of the SB.

- Respiration: The respiration affects mainly the SW activity due to its similarity in frequency (Chen et al. 1993; Lin \& Chen 1994). The origin of this interference can be due to the variation of the distance between the surface electrodes and the intestinal sources, and also due to the variation of the contact impedance between the electrodes and the skin (Ramos et al. 1993). The presence of the respiratory interference depends strongly on the recording conditions, precisely on the fixation of the contact electrodes, on the position of the electrodes and on the position of the subject in study.

- Components of very low frequency: In the external EEnG recording, it can often be observed components whose frequency is below the lowest frequency of the intestinal pacemaker (Chen et al. 1993; Garcia-Casado et al. 2005; Prats-Boluda et al. 2007). Its origin may be due to the use of an inappropriate signal conditioning and digitalization system (Mintchev et al. 2000), to the variation of the contact impedance between the surface electrodes and the skin, or to the bioelectric activity of other organs with a slower dynamics (Chen et al. 1993). In this respect, the gastric activity whose frequency is around $3 \mathrm{cpm}$ might be the principal source of the very low frequency interferences in the study of the human surface EEnG (Chen et al. 1993).

- Artifacts: The artifacts consist of abrupt changes on the amplitude of the external myoelectrical signal. Its occurrence is intermittent and unpredictable and they can completely distort the signal power spectrum (Verhagen et al. 1999). Liang et al. showed in their studies that the morphology of the artifacts in external myoelectrical recordings is diverse and depends on the kind of movement, being its amplitude in the time domain very high compared to that of the target signal (Liang et al. 1997). In addition, the presence of artifacts usually provokes a considerable increase in the spectral content, especially in the high frequency range (Liang et al. 1997).

In short, all these interferences must be somehow eliminated before the analysis of the external EEnG signal in order to be able to obtain more robust parameters that characterize the intestinal activity from the non-invasive myoelectrical recordings. 


\section{Enhancement of surface EEnG recordings}

In the past years, there have been developed diverse signal processing techniques for the interferences reduction on the biomedical signals which can be suitable for being applied to the external EEnG signals, such as adaptive filtering, independent component analysis (ICA), or empirical mode decomposition (EMD).

Given the peculiarity of the intestinal EEnG signal, i.e., that the energies of the SW activity and the SB activity are distributed in different frequency ranges, this section is divided into two subsections, one for each of these frequency bands: the low frequency band where the intestinal SW activity is contained, and high frequency band where the SB activity spreads its energy.

\subsection{Study of the EEnG in the low frequency band}

From the first studies that have validated the possibility of recording the intestinal myoelectrical activity on abdominal surface, diverse techniques have been proposed for the interferences' reduction in the low frequency range. The aim of these techniques is to cancel respiration and components of very low frequency, and to extract the intestinal SW activity contained in the external EEnG signal. The final goal is to improve the quality of the external EEnG signal and to bring the non-invasive myoelectrical techniques closer to the clinical application. Among these interferences, the respiratory interference has received special attention of diverse researchers, given its similarity in frequency with the intestinal SW activity.

\subsubsection{Adaptive filtering}

The fundamental idea of adaptive filtering is the following one: it is given a primary signal which is a mixture of the target signal and the interference, and a reference signal which can be an estimation of the interference (interference canceller structure), an estimation of the target signal (signal-enhancer structure), or an estimation of the occurrence in the time (Ferrara \& Widrow 1981). In agreement with a pre-established target function, for example the minimizations of the expected value of the output signal in the interference canceller structure, the parameters of the filter are changed by means of an adaptive algorithm. The result of this process is the obtaining of an output signal that turns out to be the best estimation of the target signal with minimal interferences content. Adaptive filtering has been widely used for the interferences' reduction contained in the biomedical signals.

With regard to the intestinal signals, diverse authors have used this technique to eliminate the respiratory interference contained in the external EEnG. Precisely, different configurations have been used: in time domain (Prats-Boluda et al. 2007); in frequency domain (Chen \& Lin 1993); and in discrete cosine transform (Lin \& Chen 1994). In the first work, the authors implemented adaptive filtering with the LMS (least minimum square) algorithm (Prats-Boluda et al. 2007). In this case, the reference signal is a filtered version of the external EEnG signal. Specifically, a band-pass filter in the respiration frequency range was used. The cut-off frequencies are obtained from the simultaneously recorded respiration signal. Figure 6 shows $120 \mathrm{~s}$ of the respiration signal (trace a) and of the external EEnG before and after the application of adaptive filtering, and their corresponding power spectral densities (PSDs). In this figure it is possible to observe that the respiratory 
interference is highly attenuated after the adaptive filtering, although a remaining component of the interference can still be observed in the processed signals.

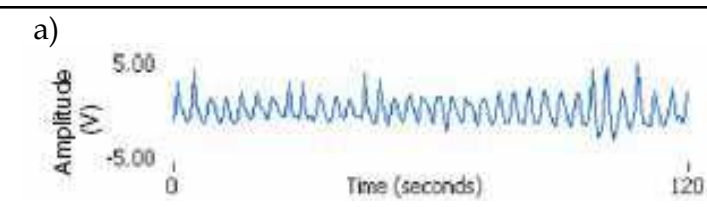

b)

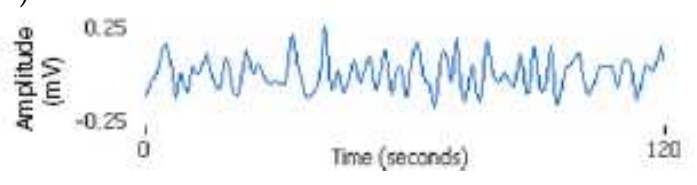

c)

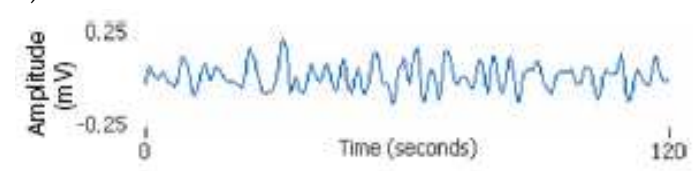

d)

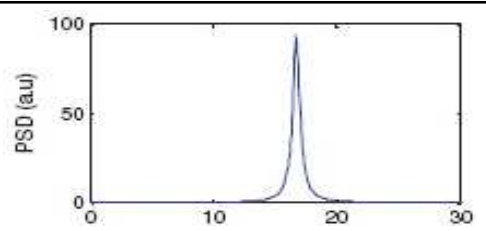

e)

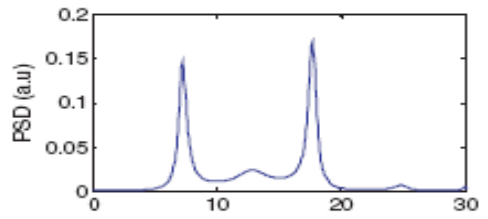

f)

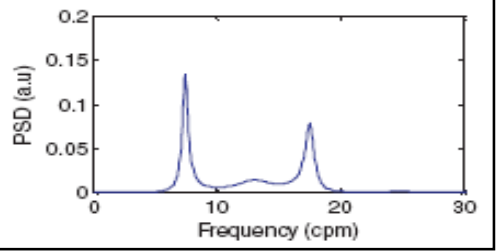

Fig. 6. a) Respiration signal. b) Original EEnG signal recorded on the human abdominal surface c) Processed signal by means of adaptive filtering. e-f) PSD of the signals that are depicted on the left-hand side (Prats-Boluda et al. 2007).

Other authors have used transform-domain adaptive filtering for the elimination of the respiratory interference from the external EEnG recording. This technique consists in applying both to the primary signal (external EEnG) and to the reference signal (respiration signal), the Fourier's transform (Chen \& Lin 1993) or the discreet cosine transform (Lin \& Chen 1994), before obtaining the target function and adjusting the filter weights. These studies concluded that the application of adaptive filtering allows improving considerably the quality of the external recording of the human EEnG. Figure 7 shows the original external EEnG signal and the filtered one by means of adaptive filtering based on the discrete cosine transform, and its corresponding PSDs in the low frequency range. In that work, the reference signal of the adaptive filter is an estimation of the target signal, which is obtained by band-pass filtering the external EEnG signal. In this figure it is possible to observe that the intestinal components $(8-12 \mathrm{cpm})$ have not been affected by the signal processing, whereas the non-desired components have been attenuated more than $20 \mathrm{~dB}$ (Lin \& Chen 1994).

The results of these studies show that the effectiveness of the adaptive filtering technique to cancel the interference strongly depends on the reference signal (Chen \& Lin 1993; Lin \& Chen 1994; Prats-Boluda et al. 2007). In this respect, the frequency of the respiratory interference contained in the surface EEnG may be identical to that of the recorded respiration signal, but the waveform and phase can be different. This can severely reduce the adaptive filtering capacity to suppress the respiratory interference of the surface EEnG, if the respiration signal is used as the reference signal in a time-domain adaptive filter. In addition, the respiratory interference is not usually present in the external EEnG recording 


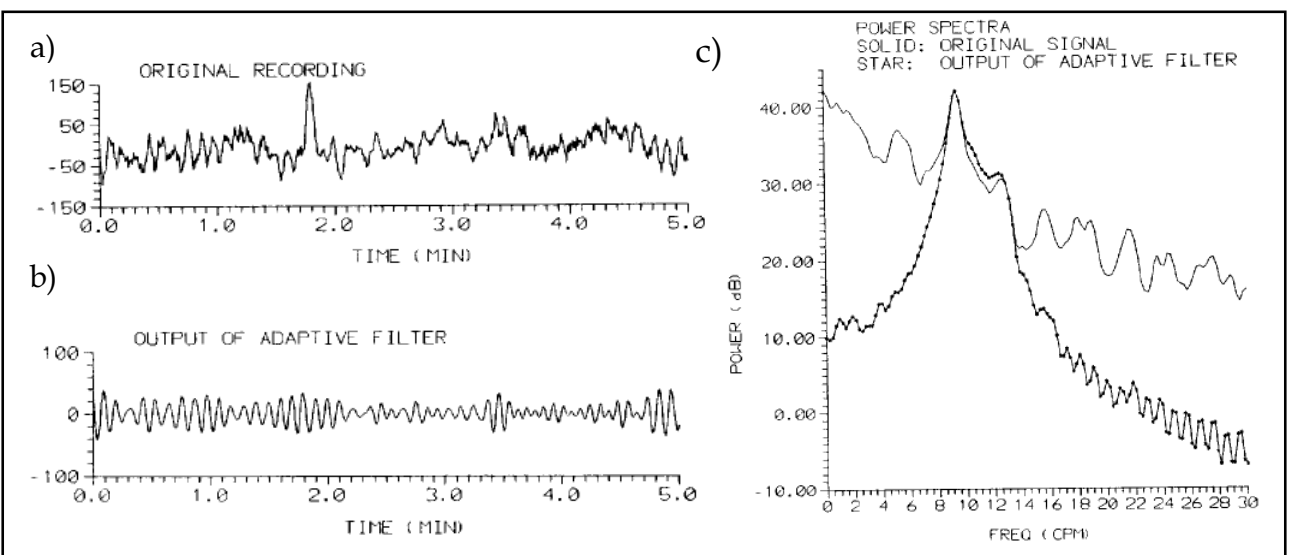

Fig. 7. a) Original external EEnG recording. b) Processed signal by means of adaptive filetering based on discrete cosine transform. c) PSD of original signal (solid line) and that of processed signal (line with stars) (Lin \& Chen 1994).

during the whole recording session. In fact, it could have great variations in its intensity for adjacent segments. This can impede the selection of the adaptive filter parameters: the order of the filter and the step size. In these cases, the extracted interference might differ from the interference which is really contained in the external myoelectrical record, and therefore the resultant signal might contain remaining interference, or the components of the target signal may be distorted (Prats-Boluda et al. 2007).

\subsubsection{Independent component analysis (ICA)}

This technique departs from the hypothesis that the observed or recorded signals are the result of an unknown mixing process of the source signals which are supposed to be mutually independent. Independent component analysis (ICA) consists in extracting a set of statistically independent components from a set of observed signals based on statistical learning of the data, without any previous knowledge of the source signals and the mixing matrix (Hyvarinen et al. 2001). In the biomedical signals context, it is usually considered that the mixing process is instantaneous and linear, assuming that the observed signals in the different channels are a simple linear combination of the attenuated source signals (James \& Hesse 2005).

The ICA algorithm has found application in diverse fields of engineering, among them the identification of the signal components and the reduction of interferences contained in the biomedical signals. To the knowledge of the authors, it has not been found any work that has used this technique to improve the quality of the external EEnG signal. Nevertheless, the results found in the literature with regard to the gastric signal, suggest us that the ICA could also be applied to the intestinal myoelectrical signals. This is the reason why the ICA has been included in the present chapter.

With respect to the myoelectrical gastric signal, the ICA has been used by diverse authors for the reduction of respiratory interference in order to recover the gastric SW activity of the external records (Liang 2001; Wang et al. 1999). These authors state that, when a few number of external EGG recordings is available, it is only possible to recover one signal of gastric 
origin in the output of the ICA algorithm, whereas the respiratory interference and other noises are concentrated in other channels (Liang 2001; Wang et al. 1999). Figure 8a-b shows an example of the application of ICA to a segment of EGG signal recorded on the human abdominal surface. Figure 8a shows 3 external channels of the original EGG record. After the application of the ICA algorithm, 3 independent components (ICs) have been obtained which can be observed in the fig. $8 \mathrm{~b}$. It is possible to appreciate that the respiration and other noises are concentrated in the channels 2 and 3 of the output, whereas the channel 1 of output, which presents less respiratory interference, corresponds to the gastric SW activity contained in the original signals. Nevertheless, the channels 2 and 3 of the output can also contain gastric SW activity. Consequently, the ICA can be a useful tool to identify the dominant frequency of the SW activity, but it is not suitable to improve the signal interference ratio of every external channel (Wang et al. 1999).

Some authors propose the identification of the gastric SW activity in each of the channels in a multichannel record of the external EGG ( 3 external channels) by means of the combined method based on ICA and adaptive filtering (Liang 2005). This combined method consists in using the output signal of the ICA algorithm, as reference signal for the implementation of adaptive filtering to each of the external channels. This technique proved to improve the quality of every channel of the external EGG (Liang 2005). This combined method also benefits from the maximum possible independence among the different output signals of the ICA algorithm, which might potentially improve the signal quality obtained by means of adaptive filtering (Liang 2005). Figure 8c shows the signals filtered by means of the combined method based in ICA and adaptive filtering. The presence of the gastric SW activity in three external channels can be observed more clearly than in the original signals.

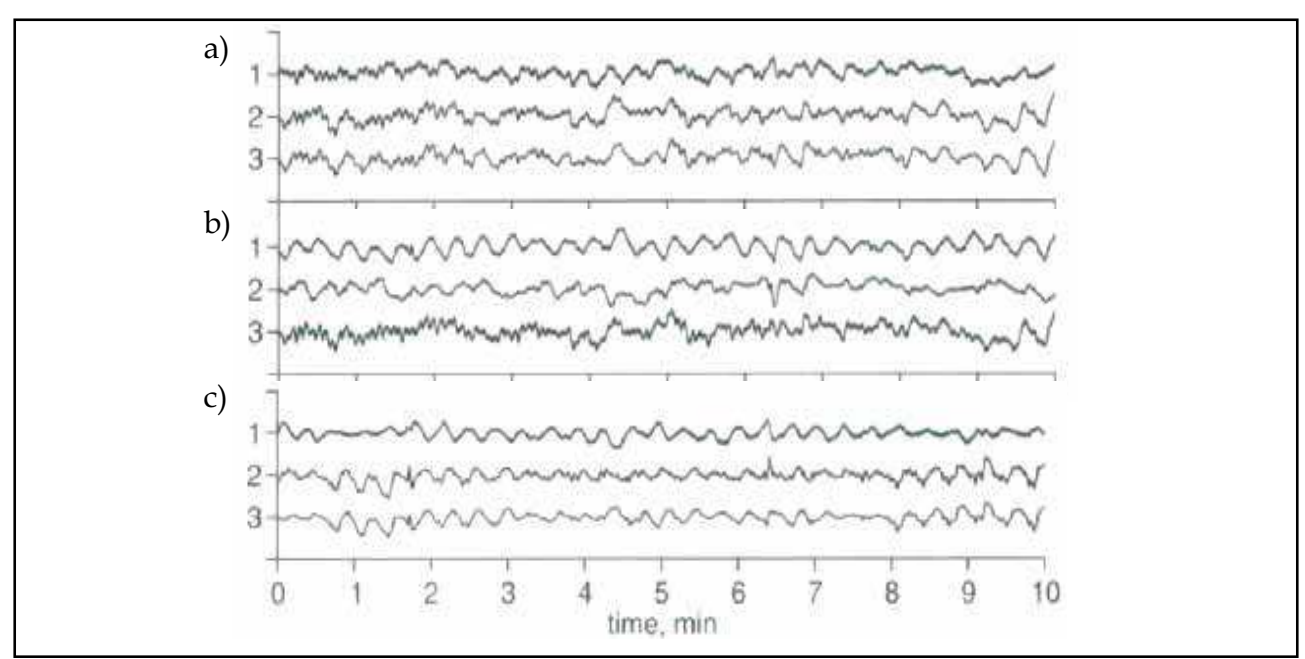

Fig. 8. Extraction of the gastric SW activity from multichannel surface EGG signal by means of a combined method based on ICA and adaptive filtering. a) 3 channels of original external EGG signals recorded simultaneously. b) Independent components estimated by the ICA algorithm (output of ICA). c) Processed signal after the application of adaptive filtering, using the channel 1 of the output of the ICA algorithm as reference signal (Liang 2005). 
The constrained ICA has also been proposed for the extraction of the gastric SW activity from the external EGG recordings (Peng et al. 2007). In this work it has been used 4 channels of external EGG, and a piezoelectric sensor placed near the navel to record the abdomen movement and the cardiac activity. The last signal will be used as reference signal to the elimination of the respiratory and cardiac interferences (Peng et al. 2007). The results of that study show that the constrained ICA allows to extract the gastric SW activity with less interference of high frequency, i.e. cardiac interference, than the conventional ICA method, thanks to the restriction of "as far as possible" to the reference signals (Peng et al. 2007).

Other authors defend that the increase of the number of simultaneously recorded channels enables improving the separability of the different components contained in the original signals (Liang 2001). In a recent study, it has been determined by means of the dynamic analysis, that a minimum of 6 simultaneously recorded channels are required for the correct separation of the different components contained in the multichannel recording of the EGG in healthy subjects (Matsuura et al. 2007). In this context, other authors who used 19 channels of surface magnetogastrogram (MGG) proved that the ICA algorithm allows the extraction of the respiratory interference, the ECG interference, the artifacts and the gastric SW activity, improving in this way the quality of the non-invasive recordings of gastric activity (Irimia \& Bradshaw 2005).

All the above mentioned works which were carried out on non-invasive recordings of gastric activity show the potential of ICA-based techniques to reduce the interferences in the low frequency range which are present in the external EEnG recordings, although to the author's knowledge, there are have not been published studies which confirm this fact. In addition, the minimal number of simultaneously recorded channels which are needed to separate the different components contained in the external EEnG signal is still to be determined. In this respect, possible future works should consider that, given the low spatial resolution of external bipolar EEnG recording, every channel might be recording the myoelectrical activity of more than one intestinal handle. This would mean that the activity of a higher number of source signals is being recorded, and therefore it might needed an even higher number of recording channels for a correct separation of the sources.

\subsubsection{Empirical mode decomposition (EMD)}

The empirical mode decomposition (EMD) algorithm was proposed initially for the study of fluid mechanics by Huang et al. (Huang et al. 1998), and soon found applications in biomedical signals processing both for the characterization of the signals and for the elimination of interferences contained in these signals (Liang et al. 2000; Maestri et al. 2007). This technique does not need any previous knowledge of the signal and it consists of expanding any complicated signal in a finite number of oscillatory functions, called intrinsic mode functions (IMFs). An IMF is defined as any function that has the same number of extrema (local maximums and minimums) as the number of zero crossings, and that has a local mean of zero (Huang et al. 1998). The IMFs defined in this way are symmetrical with regard to the zero axis, and they have a unique local frequency. This is, the different IMFs extracted from a signal do not share the same frequency at the same time (Huang et al. 1998).

The IMFs can be interpreted as adaptive basis functions which are directly extracted from the signal. Therefore, the EMD method is suitable for the analysis of the signals obtained from non-linear and non-stationary processes (Huang et al. 1998). This is a principal 
advantage of EMD over the Fourier's transform, in which the basis functions are linear combinations of sinusoidal waves. In comparison with Wavelet analysis, the IMFs obtained by the EMD method, which represent the dynamic processes masked inside the original signal, have usually better physical interpretation of the process (Huang et al. 1998).

With regard to the gastrointestinal signals, the EMD has been used for the reduction of the interferences contained in the external EGG recordings (Liang et al. 2000), and in the external EEnG recordings (Ye et al. 2007). In the latter work, the EMD method was used to analyze the external EEnG recordings obtained from anesthetized dogs (assisted respiration with mechanical ventilation fixed to $27 \mathrm{cpm}$ ), in order to reduce the interferences in the low frequency range and to improve the quality of the external EEnG signals (Ye et al. 2007).

In figure 9 it is shown an example of the application of the EMD to 1 minute of external EEnG signal. The preprocessed external EEnG signal appears in the trace a). Its corresponding PSD between 0 and $1 \mathrm{~Hz}$ (trace h) shows two clear peaks: at $0.20 \mathrm{~Hz}$ and at $0.45 \mathrm{~Hz}$. The $0.20 \mathrm{~Hz}$ component is probably associated with the intestinal SW activity, since this frequency is within the frequency range of the intestinal SW rate. The $0.45 \mathrm{~Hz}$ component probably corresponds to the respiratory interference given the coincidence with the respiration frequency $(27 \mathrm{cpm})$, and in addition it cannot be a harmonic of the intestinal SW activity. The decomposition of this signal by means of the EMD algorithm has given rise to 4 IMFs and a residual signal (traces b-f), their corresponding PSDs are depicted on the right-hand side. In these figures it is possible to observe that every IMF has different frequency components. Especially, the first extracted IMF fits to the most rapid variation of the original signal. As the process of decomposition advances, the mean frequency of the IMFs diminishes gradually. In this case, the spectral analysis has identified the $\mathrm{IMF}_{2}$ component as the respiratory interference, and the residual signal $r_{4}$ as an interference of very low frequency. Therefore, the processed signal (trace g) is obtained adding the $\mathrm{IMF}_{1}$, $\mathrm{IMF}_{3}$ and $\mathrm{IMF}_{4}$. A comparison of the original signal with the processed one, allows us to affirm that the application of the EMD method has considerably reduced the interferences in the low frequency range, making easy to identify the myoelectrical signal of intestinal origin that is contained in the original signal.

The application of the EMD method allows to improve significantly the signal-tointerference (S/I) ratio. Furthermore, this improvement owes principally to the attenuation of the energy associated with the interferences, whereas the energy associated with the target signal remains almost constant (Ye et al. 2007). Thanks to the reduction of the interferences by means of the EMD method, the variability of the dominant frequency of the external EEnG signal is also considerably diminished. These results show that the EMD method is a very helpful tool to improve the quality of the external EEnG recordings, and therefore it is possible to extract more trustworthy parameters that permit to identify noninvasively the intestinal SW activity. Nevertheless, this study still presents some limitations, for example , the respiration is assisted and fixed $(0.45 \mathrm{~Hz})$ (Ye et al. 2007). When recording in physiological conditions, the respiration frequency might change during the session which could complicate the identification of the respiratory interference in the different IMFs obtained from the EMD algorithm. In this respect, the simultaneous recording of the respiration signal would be of great help in order to obtain a reference of the breathing frequency, and hence to correctly identify and eliminate this interference on the external signal. Also, the applicability of the EMD method to the human external EEnG recordings in physiological conditions has to be checked in future studies. 


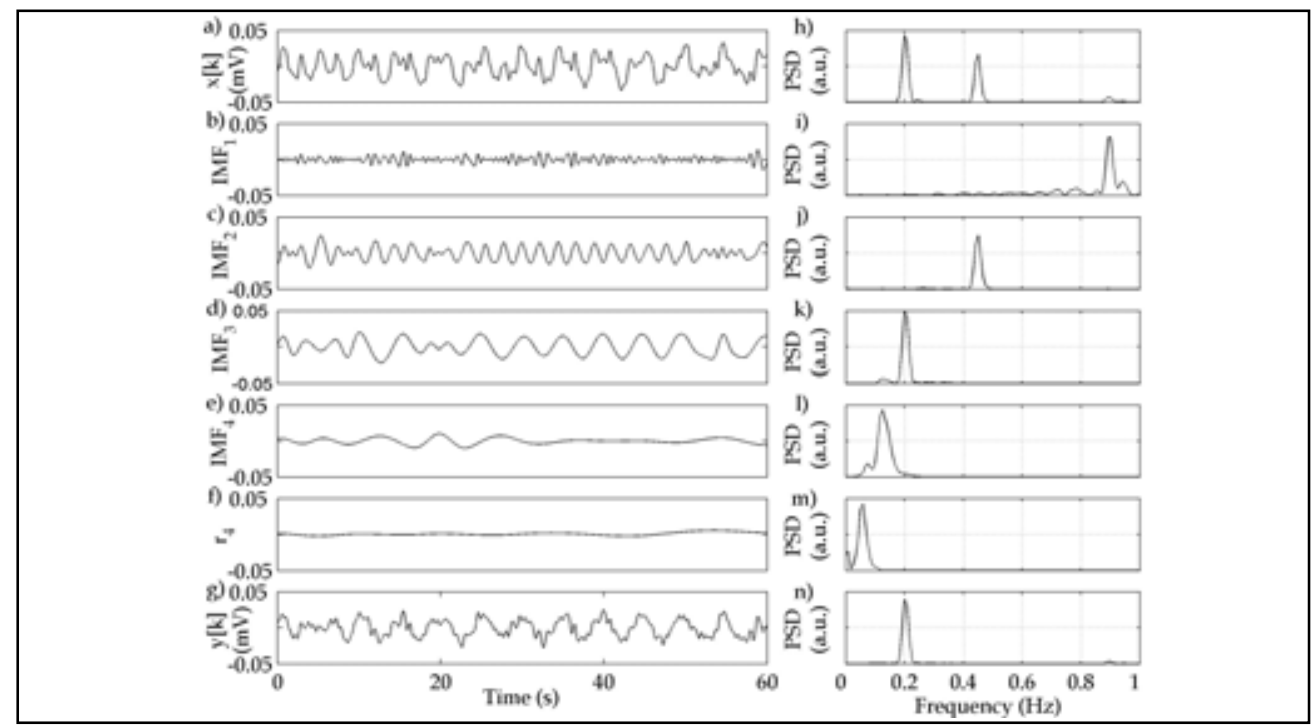

Fig. 9. Application of the EMD method to 1 minute of surface EEnG recording with strong respiration interference $(0.45 \mathrm{~Hz})$. a) Original EEnG recording after preprocessing $x[\mathrm{k}]$ (low pass filter with cut-off frequency at $2 \mathrm{~Hz}$ ). b-f). b-f) Outputs of the EMD method: four IMFs and one residual signal. g) Processed signal y[k]: sum of $\mathrm{IMF}_{1}, \mathrm{IMF}_{3}$ y IMF $\mathrm{IM}_{4}$ h-n) PSD of the signals that are depicted on the left-hand side (Ye et al. 2007). The PSD of the processed signal is represented at the same scale of the original signal

\subsection{Study of the EEnG in the high frequency band}

Given the small amplitude of the intestinal SB activity when recorded on abdominal surface, and the strong interferences that are present in the high frequency range which mainly are the ECG interference and movement artifacts, several techniques have been developed for the reduction of these interferences. These interferences should be removed so as to improve the quality of the external EEnG signal for the correct identification and quantification of the SB activity in a non-invasive way. It should be emphasized here that very few works have been found that are related to the reduction of these interferences (ECG and artifacts) in the intestinal signal, since the majority of the authors have focused their studies on the intestinal SW activity and in these cases the high frequency interferences can be eliminated by conventional low-pass filtering (Bradshaw et al. 1997; Chen \& Lin 1993; Lin \& Chen 1994; Seidel et al. 1999).

\subsubsection{Adaptive filtering}

Adaptive filtering has been used for the reduction of the ECG interference contained in the canine external EEnG recording (Garcia-Casado et al. 2006). In that study, a technique based on synchronized averaging has been used to estimate the ECG interference of the external EEnG. Precisely, the interference estimator is obtained by averaging a number of windows of the external EEnG recording using the onset of the R wave of the ECG as synchronizing event. This procedure is similar to the obtaining of event-related potentials. Once the 
estimation of ECG's interference is obtained, it is used as the reference signal for the implementation of an adaptive filter based on the LMS algorithm for the elimination of this interference (Garcia-Casado et al. 2006).

Figure 10 shows 10s of the original external EEnG signal and after being processed by means of the adaptive filter in a period of rest (traces a and b), and in a period of maximum contractile activity (traces $\mathrm{c}$ and d). In these figures it can be observed that the application of the adaptive filter allows reducing the ECG interference contained in the external EEnG signal both in periods of rest and of maximum contractile activity; whereas both components of the myoelectrical intestinal activity (SW and SB) are minimally affected by the signal processing (Garcia-Casado et al. 2006). The reduction of the interference has enabled improving the ECG's signal-to-interference ratio significantly (Garcia-Casado et al. 2006). These results confirm that adaptive filtering can be a tool of great help to reduce ECG interference and to improve the quality of the external EEnG recordings.

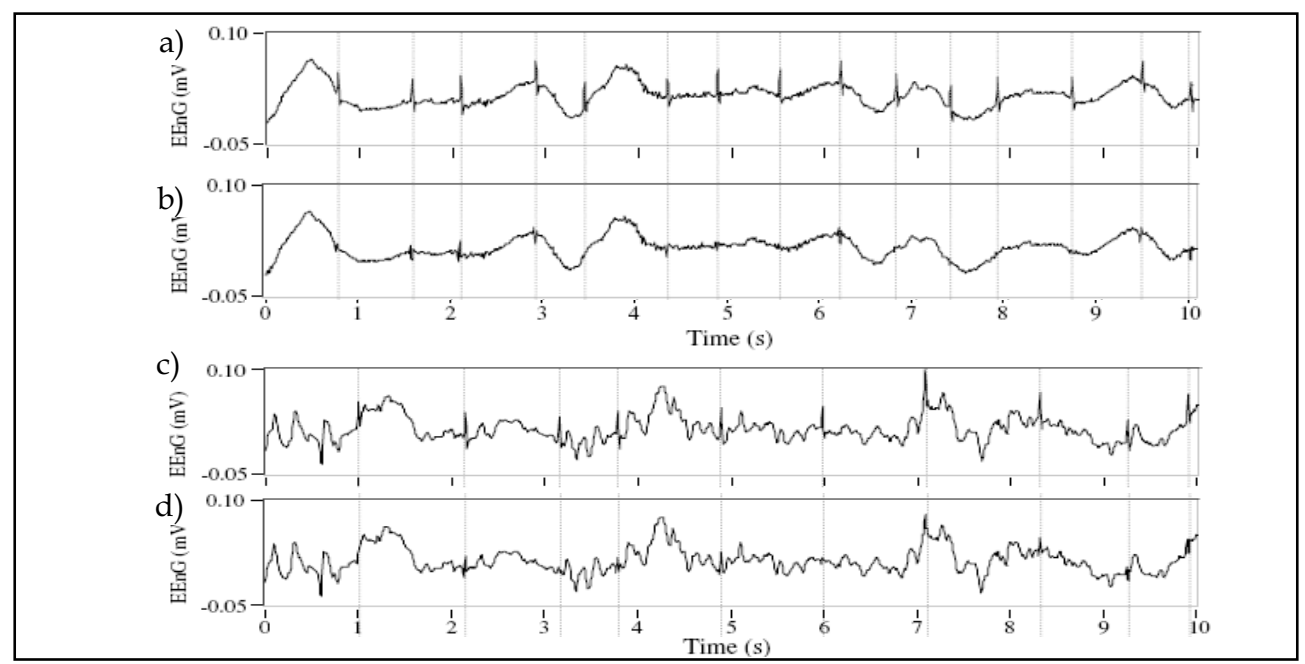

Fig. 10. a-b) Original external EEnG signal during a period of rest and the processed signal by adaptive filtering respectively. c-d) Original external EEnG signal during a period of maximum contractile activity and after being processed by adaptive filtering respectively. (Garcia-Casado et al. 2006). Signals were recorded from conscious dogs in fasting state. Fiducial points of the R-wave are marked with a vertical broken line.

\subsubsection{Combined method based on EMD and ICA}

In a recent study, a combined method based on EMD and ICA has been proposed to reduce both the ECG interference and the movement artifacts in the high frequency range of the multichannel recordings of the external EEnG (Ye et al. 2008). This combined method consists in firstly analyzing separately each of 4 simultaneous recordings of external EEnG by means of the EMD algorithm. Later, there are selected those IMFs (results of the EMD algorithm) whose mean frequency is revealed to be higher than $1 \mathrm{~Hz}$ by means of spectral analysis. This procedure usually results in a variable number of IMFs which contain the information of high frequency components $(>1 \mathrm{~Hz})$. These IMFs obtained from 4 external channels will be analyzed together by means of the ICA algorithm in order to obtain the 
independent components (Ye et al. 2008). Subsequently, the interferences associated to ECG and movement artifacts are identified in the outputs of the ICA algorithm. Finally, the processed signals are reconstructed without the identified interferences by means of an inverse process.

In figure 11 it is shown an example of the application of the combined method to a window of external EEnG signals in a period of rest. In the original signals of external EEnG recordings (traces b-e), it can be observed a low frequency component (3-4 cycles in $15 \mathrm{~s}$ ) which is associated with the intestinal SW activity. It can also be appreciated the presence of strong ECG interference in the channels 1 and 2 (traces $b$ and c), which is synchronized with the simultaneously recorded ECG signal (trace a). On the other hand, the ECG interference in the channels 3 and 4 (traces $\mathrm{d}$ and e) is weak. Finally, it can also be appreciated in the original signals the appearance of movement artifacts in the 4 external channels around the second 10. The signals processed by means of the combined method are shown in traces g-j. A comparison of the original signals with the processed ones allows deducing that the application of the combined method has cancelled both ECG interference and movement artifacts from the original signals, without affecting the intestinal myoelectrical activity.

The application of the combined method to a window of external EEnG signals in a period of maximal contractile activity appears in figure 12. Again, it can be observed the presence of a low frequency activity in the 4 external channels (traces b-e), that probably corresponds to the intestinal SW activity. In these traces it is also possible to observe the presence of components of high frequency and low amplitude which are superposed to the intestinal SW activity, which are possibly associated with the intestinal SB activity. The appearance of these components of high frequency impedes the visual identification of ECG interference in the external EEnG signal. In this case, the ECG interference can only be clearly appreciated in the channel 2 (trace c). The signals processed by means of the combined method are shown in traces g-j. Again, the application of the combined method has eliminated the ECG interference contained in the original signals, whereas the intestinal myoelectrical activity has been minimally affected.
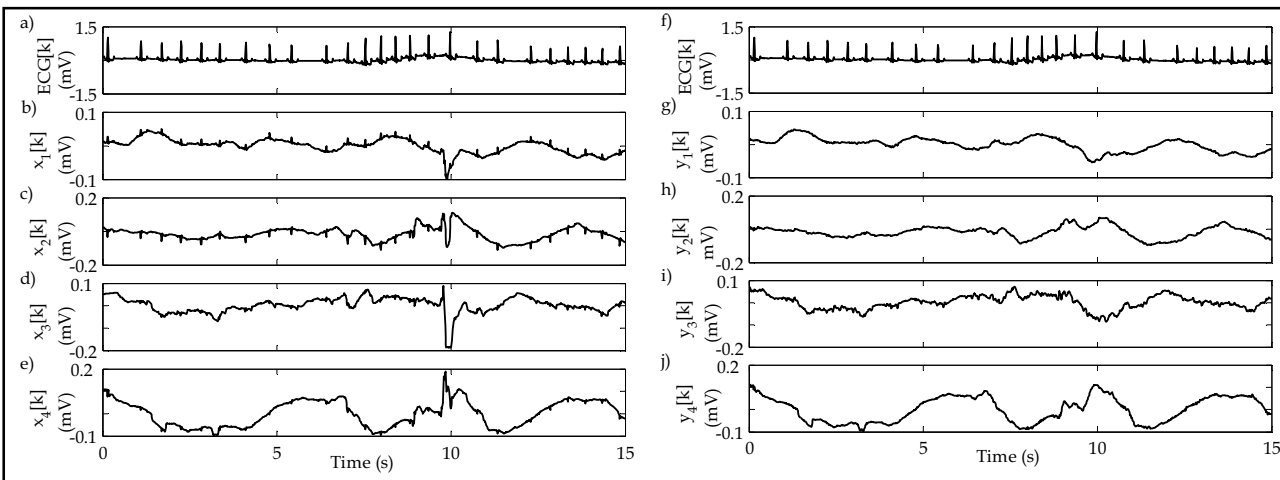

Fig. 11. Application of the combined method based on EMD and ICA to multichannel surface EEnG recording; the window length of the analysis is 30s. a) and f) ECG signal. b-e) original signals of 4 surface EEnG channels $\left(x_{1}[k]-x_{4}[k]\right)$ during a period of rest. It can be appreciated the appearance of movement artifacts around the second 10. g-j) Processed signals $y_{1}[k]-y_{4}[k]$. Signals were recorded from conscious dogs in fasting state. 

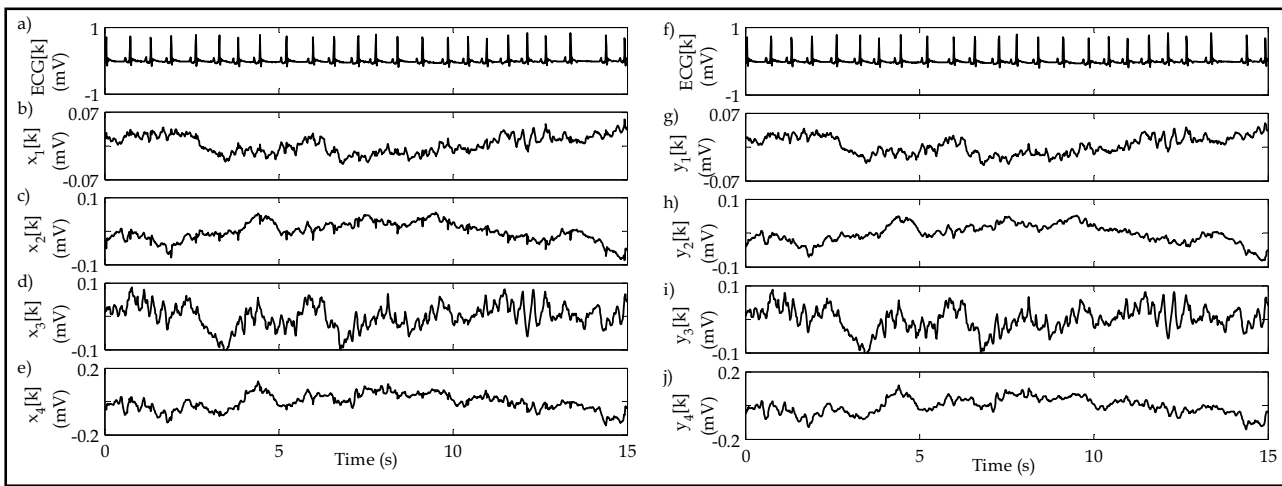

Fig. 12. Application of the combined method based on EMD and ICA to multichannel surface EEnG recording; the window length of the analysis is 30s. a) and f) ECG signal. b-e) original signals of 4 surface EEnG channels $\left(x_{1}[k]-x_{4}[k]\right)$ during a period of maximum contactile activity. g-j) Processed signals $y_{1}[k]-y_{4}[k]$. Signals were recorded from conscious dogs in fasting state.

The results of that study indicate that the application of the combined method allows to significantly improve the signal-to- ECG interference of the external EEnG recordings, and to reduce the variability of the non-invasive indicator of the intestinal motility (Ye et al. 2008). This is due to the fact that the combined method enables achieving an improvement on the separation of the different components contained in the original signal when compared to the conventional ICA method. The difference between both techniques lies in the reduction of the number of sources which are present in the original signals by restricting the frequency band of analysis (over $1 \mathrm{~Hz}$ ), and also by using a higher number of virtual channels due to the decomposition of the original signals into multiple oscillatory functions using the EMD algorithm. When compared to conventional EMD method, if only EMD was used, the SB activity could be mixed with interferences of similar instant frequencies in the same IMFs, whereas the combined method takes advantage of the capacity of the ICA algorithm to separate these independent components. This preliminary study shows the potential of the use of the combined method based on EMD and ICA to improve the quality of the external EEnG recording. The application of this method permits to obtain more robust non-invasive parameters which measure the internal intestinal motility.

\section{Futures perspectives}

The results from recent works suggest the possibility of detecting both components of the intestinal myoelectrical activity in the external recording of EEnG in animal model (GarciaCasado et al. 2005; Ye et al. 2008). Future studies on animal models might test the possibility of the non-invasive myoelectrical techniques to diagnose different pathologies related to intestinal activity dysfunctions. On the other hand, other recent studies suggest the possibility of recording the human gastric $\mathrm{SB}$ activity in the external recordings of the magnetogastrogram (Irimia et al. 2006). Based on these works, we believe that the intestinal $\mathrm{SB}$ activity might also be detected in the external myoelectrical records of humans. Future 
researches should extend the analysis of the external EEnG signals from humans out of the range of the intestinal SW activity, and to focus their efforts on the frequency band of the intestinal SB activity in order to check the possibility of detecting not only the pacemaker activity but also the contractile activity on abdominal surface of humans.

In this chapter, it has been presented a review on the different techniques used for the elimination of interferences contained in the EEnG external recordings. Among them, it has to be outlined the EMD method to cancel the interferences in the low frequency range, and the combined method based on EMD and ICA to reduce the interferences in the high frequency range. The analysis of quantitative parameters which allow evaluating the reduction of these interferences, has validated the applicability of these techniques to improve the quality of the canine external EEnG. By means of the application of these techniques, more robust parameters of the intestinal activity from the external recordings can be obtained. Specifically, it diminishes considerably the variability of the dominant frequency and of the intestinal motility index. The previously mentioned signal processing techniques could be easily adapted to be applied to the non-invasive recordings of the intestinal myoelectrical activity from humans. Fundamentally the frequency bands should be adjusted to the human EEnG characteristics. All this, in order to bring the non-invasive myoelectrical techniques closer to their future clinical application.

Besides the development of signal processing techniques, which turns out to be indispensable to improve the quality of the external EEnG, different research groups are developing techniques to record the Laplacian of the potential so as to improve the spatial resolution of conventional bipolar and monopolar recordings ( $\mathrm{Li}$ et al. 2005; Prats-Boluda et al. 2007). Theoretically, the Laplacian of the potential is proportional to the second derivative of the orthogonal current density to the surface of the body (He \& Cohen 1992). The Laplacian technique could be considered to be similar to a filter that assigns higher weights to the orthogonal bioelectric dipoles adjacent to the measuring surface, and attenuates the bioelectrical interferences which propagate tangentially to the abdominal surface (He \& Cohen 1992). Recent studies have demonstrated that the signal-to-ECG interference ratio of the discrete approximation to the Laplacian recording of the EEnG is significantly higher than that of bipolar EEnG recordings (Prats-Boluda et al. 2007). At present, active electrodes which obtain a direct estimation of the Laplacian potential by concentric rings are being developed. The use of these Laplacian electrodes would improve the spatial resolution of the non-invasive recordings of the intestinal myoelectrical activity. These recordings, together with the above mentioned signal processing techniques, would permit to derive more robust non-invasive parameters that characterize the intestinal SW and SB activity.

Finally, the development of pattern classifiers which enable discriminating with better accuracy physiological and pathological conditions from myoelectrical recordings is another key point for the future clinical application of this technique. In respect to this, the application of neural networks and support vector machines to the external EGG signals has demonstrated its utility to detect delayed gastric emptying (Chen et al. 2000; Liang \& Lin 2001). Future studies should concentrate in adapting these pattern classifiers to distinguish the external EEnG signals in different pathological conditions from healthy conditions. 


\section{Conclusion}

Both the SW activity and the intestinal SB activity can be recorded on the abdominal surface, which suggests that the EEnG recordings on the abdominal surface would be an alternative method for the non-invasive monitoring of the intestinal activity. Nevertheless, the external EEnG signal is very weak, and in addition it is contaminated by a set of interferences (ECG, artifacts, respiration and components of very low frequency). The presence of these interferences impedes the extraction and interpretation of parameters that characterize the intestinal myoelectrical activity based on its non-invasive record. In this respect, the application of modern signal processing techniques turns out to be indispensable to reduce these interferences, and to improve the quality of the external recordings of the EEnG. In parallel, advances in signal recording and instrumentation techniques like the Laplacian recording of the potential also might contribute to the enhancement of the raw EEnG external signals, by permitting to obtain external signals with less physiological interference and with better spatial resolution. Thanks to the development of the signal processing techniques and to the improvement in the instrumentation techniques, it is possible to obtain robust parameters of the intestinal SW and SB activity derived from the surface EEnG recordings that bring these non-invasive myoelectrical techniques closer to their clinical application.

\section{References}

Akin, A. \& Sun, H. H. (1999), Time-frequency methods for detecting spike activity of stomach, Med. Biol. Eng Comput., vol. 37, No. 3, pp. 381-390, ISBN. 0140-0118.

An, Y. J., Lee, H., Chang, D., Lee, Y., Sung, J. K., Choi, M., \& Yoon, J. (2001), Application of pulsed Doppler ultrasound for the evaluation of small intestinal motility in dogs, $J$. Vet. Sci., vol. 2, No. 1, pp. 71-74.

Atanassova, E., Daskalov, I., Dotsinsky, I., Christov, I., \& Atanassova, A. (1995), Noninvasive Electrogastrography .2. Human Electrogastrogram, Archives of Physiology and Biochemistry, vol. 103, No. 4, pp. 436-441, ISBN. 1381-3455.

Bass, P. \& Wiley, J. N. (1965), Electrical and Extraluminal Contractile-Force Activity of Duodenum of Dog, Am. J. Dig. Dis., vol. 10, No. 3, pp. 183-200, ISBN. 0002-9211.

Bradshaw, L. A., Allos, S. H., Wikswo, J. P., \& Richards, W. O. (1997), Correlation and comparison of magnetic and electric detection of small intestinal electrical activity, Am. J. Physiol. -Gastroint. Liver Physiol., vol. 35, No. 5, p. G1159-G1167, ISBN. 01931857.

Brown, B. H., Smallwood, R. H., Duthie, H. L., \& Stoddard, C. J. (1975), Intestinal SmoothMuscle Electrical Potentials Recorded from Surface Electrodes, Medical \& Biological Engineering, vol. 13, No. 1, pp. 97-103, ISBN. 0025-696X.

Byrne, K. G. \& Quigley, E. M. M. (1997), Antroduodenal manometry: An evaluation of an emerging methodology, Dig. Dis., vol. 15, pp. 53-63, ISBN. 0257-2753.

Camilleri, M., Hasler, W. L., Parkman, H. P., Quigley, E. M. M., \& Soffer, E. (1998), Measurement of gastrointestinal motility in the GI laboratory, Gastroenterology, vol. 115, No. 3, pp. 747-762, ISBN. 0016-5085.

Chang, F. Y., Lu, C. L., Chen, C. Y., Luo, J. C., Lee, S. D., Wu, H. C., \& Chen, J. Z. (2007), Fasting and postprandial small intestinal slow waves non-invasively measured in subjects with total gastrectomy, J Gastroenterol. Hepatol., vol. 22, No. 2, pp. 247-252. 
Chen, J. D. \& Lin, Z. (1993), Adaptive cancellation of the respiratory artifact in surface recording of small intestinal electrical activity, Comput. Biol. Med., vol. 23, No. 6, pp. 497-509.

Chen, J. D., Lin, Z., \& Mccallum, R. W. (2000), Noninvasive feature-based detection of delayed gastric emptying in humans using neural networks, IEEE Trans. Biomed. Eng, vol. 47, No. 3, pp. 409-412.

Chen, J. D., Richards, R. D., \& Mccallum, R. W. (1994), Identification of Gastric Contractions from the Cutaneous Electrogastrogram, American Journal of Gastroenterology, vol. 89, No. 1, pp. 79-85, ISBN. 0002-9270.

Chen, J. D., Schirmer, B. D., \& Mccallum, R. W. (1993), Measurement of Electrical-Activity of the Human Small-Intestine Using Surface Electrodes, IEEE Trans. Biomed. Eng., vol. 40, No. 6, pp. 598-602, ISBN. 0018-9294.

Chen, J. D., Vandewalle, J., Sansen, W., Vantrappen, G., \& Janssens, J. (1990), Adaptive Spectral-Analysis of Cutaneous Electrogastric Signals Using Autoregressive Moving Average Modeling, Med. Biol. Eng Comput., vol. 28, No. 6, pp. 531-536, ISBN. 0140-0118.

Delvaux, M. (2003), Functional bowel disorders and irritable bowel syndrome in Europe, Aliment. Pharmacol. Ther., vol. 18 Suppl 3, pp. 75-79.

Diamant, N. E. \& Bortoff, A. (1969), Nature of the intestinal low-wave frequency gradient, Am. J. Physiol., vol. 216, No. 2, pp. 301-307.

El-Murr, M., Kimura, K., Ellsberg, D., Yamazato, M., Yoshino, H., \& Soper, R. T. (1994), Motility of isolated bowel segment Iowa model III, Dig. Dis. Sci., vol. 39, No. 12, pp. 2619-2623.

Ferrara, E. R. \& Widrow, B. (1981), Multichannel Adaptive Filtering for Signal Enhancement, IEEE Trans. Acoustics Speech and Signal Processing, vol. 29, No. 3, pp. 766-770, ISBN. 0096-3518.

Garcia-Casado, J., Martinez-de-Juan, J. L., \& Ponce, J. L. (2006), Adaptive filtering of ECG interference on surface EEnGs based on signal averaging, Physiol. Meas., vol. 27, No. 6, pp. 509-527, ISBN. 0967-3334.

Garcia-Casado, J., Martinez-de-Juan, J. L., \& Ponce, J. L. (2005), Noninvasive measurement and analysis of intestinal myoelectrical activity using surface electrodes, IEEE Trans. Biomed. Eng., vol. 52, No. 6, pp. 983-991.

Garcia-Casado, J., Martinez-de-Juan, J. L., Silvestre, J., Saiz, J., \& Ponce, J. L. (2002), Identification of surface recordings of electroenterogram through time-frequency analysis, 4th International Workshop on Biosignal Interpretation, Como, Italy.

He, B. \& Cohen, R. J. (1992), Body surface Laplacian ECG mapping, IEEE Trans. Biomed. Eng, vol. 39, No. 11, pp. 1179-1191.

Horowitz, B., Ward, S. M., \& Sanders, K. M. (1999), Cellular and molecular basis for electrical rhythmicity in gastrointestinal muscles, Annu. Rev. Physiol, vol. 61, pp. 1943.

Huang, N. E., Shen, Z., Long, S. R., Wu, M. L. C., Shih, H. H., Zheng, Q. N., Yen, N. C., Tung, C. C., \& Liu, H. H. (1998), The empirical mode decomposition and the Hilbert spectrum for nonlinear and non-stationary time series analysis, Proc. Roy. Soc. LOND A MAT, vol. 454, No. 1971, pp. 903-995, ISBN. 1364-5021.

Hyvarinen, A., Karhunen, J., \& Oja, E. (2001), Independent component analysis, New York: John Wiley \& Sons. 
Irimia, A. \& Bradshaw, L. A. (2005), Artifact reduction in magnetogastrography using fast independent component analysis, Physiol. Meas., vol. 26, No. 6, pp. 1059-1073.

Irimia, A., Richards, W. O., \& Bradshaw, L. A. (2006), Magnetogastrographic detection of gastric electrical response activity in humans, Physics in Medicine and Biology, vol. 51, No. 5, pp. 1347-1360, ISBN. 0031-9155.

James, C. J. \& Hesse, C. W. (2005), Independent component analysis for biomedical signals, Physiol Meas., vol. 26, No. 1, p. R15-R39, ISBN. 0967-3334.

Lausen, M., Reichenbacher, D., Ruf, G., Schoffel, U., \& Pelz, K. (1988), Myoelectric activity of the small bowel in mechanical obstruction and intra-abdominal bacterial contamination, Eur. Surg. Res., vol. 20, No. 5-6, pp. 304-309.

Levy, J., Harris, J., Chen, J., Sapoznikov, D., Riley, B., De La, N. W., \& Khaskelberg, A. (2001), Electrogastrographic norms in children: toward the development of standard methods, reproducible results, and reliable normative data, J. Pediatr. Gastroenterol. Nutr., vol. 33, No. 4, pp. 455-461.

Li, G., Wang, Y., Lin, L., Jiang, W., Wang, L. L., Lu, C., \& Besio, W. G. (2005), Active Laplacian Electrode for the data-acquisition system of EHG, Journal of Physics: Conference series., vol. 13, pp. 330-335.

Liang, H. L. (2001), Adaptive independent component analysis of multichannel electrogastrograms, Med. Eng. Phys., vol. 23, No. 2, pp. 91-97, ISBN. 1350-4533.

Liang, H. L. (2005), Extraction of gastric slow waves from electrogastrog rams: combining independent component analysis and adaptive signal enhancement, Med. Biol. Eng. Comput., vol. 43, No. 2, pp. 245-251, ISBN. 0140-0118.

Liang, H. L. \& Lin, Z. (2001), Detection of delayed gastric emptying from electrogastrograms with support vector machine, IEEE Trans. Biomed. Eng, vol. 48, No. 5, pp. 601-604.

Liang, H. L., Lin, Z., \& Mccallum, R. W. (2000), Artifact reduction in electrogastrogram based on empirical mode decomposition method, Med. Biol. Eng. Comput., vol. 38, No. 1, pp. 35-41.

Liang, J., Cheung, J. Y., \& Chen, J. D. Z. (1997), Detection and deletion of motion artifacts in electrogastrogram using feature analysis and neural networks, Ann. Biomed. Eng., vol. 25, No. 5, pp. 850-857, ISBN. 0090-6964.

Lin, Z. Y. \& Chen, J. D. Z. (1994), Recursive Running DCT Algorithm and Its Application in Adaptive Filtering of Surface Electrical Recording of Small-Intestine, Med. Biol. Eng. Comput., vol. 32, No. 3, pp. 317-322, ISBN. 0140-0118.

Maestri, R., Pinna, G. D., Porta, A., Balocchi, R., Sassi, R., Signorini, M. G., Dudziak, M., \& Raczak, G. (2007), Assessing nonlinear properties of heart rate variability from short-term recordings: are these measurements reliable?, Physiol Meas., vol. 28, No. 9, pp. 1067-1077.

Martinez-de-Juan, J. L., Saiz, J., Meseguer, M., \& Ponce, J. L. (2000), Small bowel motility: relationship between smooth muscle contraction and electroenterogram signal, Med. Eng. Phys., vol. 22, No. 3, pp. 189-199.

Matsuura, Y., Yokoyama, K., Takada, H., \& Shimada, K. (2007), Dynamics analysis of electrogastrography using Double-Wayland algorithm, Conf Proc.IEEE Eng Med.Biol.Soc., pp. 1973-1976.

Mintchev, M. P. \& Bowes, K. L. (1996), Extracting quantitative information from digital electrogastrograms, Med. Biol. Eng. Comput., vol. 34, No. 3, pp. 244-248, ISBN. 01400118. 
Mintchev, M. P., Rashev, P. Z., \& Bowes, K. L. (2000), Misinterpretation of human electrogastrograms related to inappropriate data conditioning and acquisition using digital computers, Dig. Dis. Sci., vol. 45, No. 11, pp. 2137-2144.

Moreno-Vazquez, J. J., Martinez-de-Juan, J. L., Garcia-Casado, J., \& Ponce, J. L. (2003), Autoregressive Spetral Analysis of Electroenterogram (EEnG) for Basic Electric Rhythm Identification, Conf.Proc.IEEE Eng Med.Biol.Soc., pp. 2539-2542 Cancun, México.

Peng, C., Qian, X., \& Ye, D. T. (2007), Electrogastrogram extraction using independent component analysis with references, Neural comput. \& Applic., vol. 16, No. 6, pp. 581-587.

Prats-Boluda, G., Garcia-Casado, J., Martinez-de-Juan, J. L., \& Ponce, J. L. (2007), Identification of the slow wave component of the electroenterogram from laplacian abdomianl surface recording in Humans, Physiol. Meas., vol. 28, pp. 1-19.

Quigley, E. M. (1996), Gastric and small intestinal motility in health and disease, Gastroenterol. Clin. North Am., vol. 25, No. 1, pp. 113-145.

Ramos, J., Vargas, M., Fernández, M., Rosell, J., \& Pallás-Areny, R. (1993), A system for monitoring pill electrode motion in esophageal ECG, Conf Proc. IEEE Eng Med.Biol.Soc., pp. 810-811 San Diego.

Seidel, S. A., Bradshaw, L. A., Ladipo, J. K., Wikswo, J. P., Jr., \& Richards, W. O. (1999), Noninvasive detection of ischemic bowel, J. Vasc. Surg., vol. 30, No. 2, pp. 309-319.

Szurszewski, J. H. (1969), A Migrating Electric Complex of the Canine Small Intestine, Am. J. Physiol. pp. 1757-1763.

Tomomasa, T., Morikawa, A., Sandler, R. H., Mansy, H. A., Koneko, H., Masahiko, T., Hyman, P. E., \& Itoh, Z. (1999), Gastrointestinal sounds and migrating motor complex in fasted humans, Am. J. Gastroenterol., vol. 94, No. 2, pp. 374-381, ISBN. 0002-9270.

Van Felius, I. D., Akkermans, L. M., Bosscha, K., Verheem, A., Harmsen, W., Visser, M. R., \& Gooszen, H. G. (2003), Interdigestive small bowel motility and duodenal bacterial overgrowth in experimental acute pancreatitis, Neurogastroenterol. Motil., vol. 15, No. 3, pp. 267-276.

Verhagen, M. A. M. T., Van Schelven, L. J., Samsom, M., \& Smout, A. J. P. M. (1999), Pitfalls in the analysis of electrogastrographic recordings, Gastroenterology, vol. 117, No. 2, pp. 453-460, ISBN. 0016-5085.

Wang, Z. S., Cheung, J. Y., \& Chen, J. D. Z. (1999), Blind separation of multichannel electrogastrograms using independent component analysis based on a neural network, Med. Biol. Eng. Comput., vol. 37, No. 1, pp. 80-86, ISBN. 0140-0118.

Weisbrodt, N. W. (1987), Motility of the small intestine, in Physiology of the Gastrointestinal Tract (Vol.1),pp. 631-633, Raven Press, New York.

Ye, Y., Garcia-Casado, J., Martinez-de-Juan, J. L., Alvarez Martinez, D., \& Prats-Boluda, G. (2008), Quantification of Combined Method for Interferences Reduction in Multichannel Surface Electroenterogram, Conf.Proc.IEEE Eng Med.Biol.Soc., pp. 3612-3615 Vancouver, Canada.

Ye, Y., Garcia-Casado, J., Martinez-de-Juan, J. L., \& Ponce, J. L. (2007), Empirical mode decomposition: a method to reduce low frequency interferences from surface electroenterogram, Med. Biol. Eng Comput., vol. 45, No. 6, pp. 541-551. 


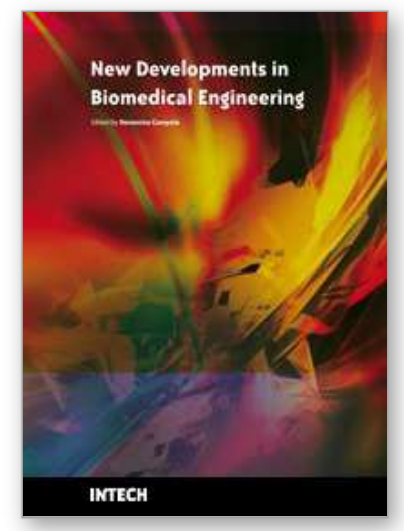

\author{
New Developments in Biomedical Engineering \\ Edited by Domenico Campolo
}

ISBN 978-953-7619-57-2

Hard cover, 714 pages

Publisher InTech

Published online 01, January, 2010

Published in print edition January, 2010

Biomedical Engineering is a highly interdisciplinary and well established discipline spanning across engineering, medicine and biology. A single definition of Biomedical Engineering is hardly unanimously accepted but it is often easier to identify what activities are included in it. This volume collects works on recent advances in Biomedical Engineering and provides a bird-view on a very broad field, ranging from purely theoretical frameworks to clinical applications and from diagnosis to treatment.

\title{
How to reference
}

In order to correctly reference this scholarly work, feel free to copy and paste the following:

Y. Ye-Lin, J. Garcia-Casado, Jose-M. Bueno-Barrachina, J. Guimera-Tomas, G. Prats-Boluda and J.L. Martinez-de-Juan (2010). Characterization and Enhancement of Non-Invasive Recordings of Intestinal Myoelectrical Activity, New Developments in Biomedical Engineering, Domenico Campolo (Ed.), ISBN: 978953-7619-57-2, InTech, Available from: http://www.intechopen.com/books/new-developments-in-biomedicalengineering/characterization-and-enhancement-of-non-invasive-recordings-of-intestinal-myoelectrical-activity

\section{INTECH}

open science | open minds

\author{
InTech Europe \\ University Campus STeP Ri \\ Slavka Krautzeka 83/A \\ 51000 Rijeka, Croatia \\ Phone: +385 (51) 770447 \\ Fax: +385 (51) 686166 \\ www.intechopen.com
}

\author{
InTech China \\ Unit 405, Office Block, Hotel Equatorial Shanghai \\ No.65, Yan An Road (West), Shanghai, 200040, China \\ 中国上海市延安西路65号上海国际贵都大饭店办公楼 405 单元 \\ Phone: +86-21-62489820 \\ Fax: +86-21-62489821
}


(C) 2010 The Author(s). Licensee IntechOpen. This chapter is distributed under the terms of the Creative Commons Attribution-NonCommercial-ShareAlike-3.0 License, which permits use, distribution and reproduction for non-commercial purposes, provided the original is properly cited and derivative works building on this content are distributed under the same license. 